\title{
ALMA OBSERVATIONS OF THE DEBRIS DISK AROUND THE YOUNG SOLAR ANALOG HD 107146
}

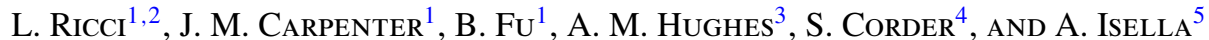 \\ ${ }^{1}$ Department of Astronomy, California Institute of Technology, MC 249-17, Pasadena, CA 91125, USA; 1ricci@ astro.caltech.edu \\ ${ }^{2}$ Harvard-Smithsonian Center for Astrophysics, 60 Garden Street, Cambridge, MA 02138, USA \\ ${ }^{3}$ Department of Astronomy, Wesleyan University, Van Vleck Observatory, 96 Foss Hill Drive, Midletown, CT 06457, USA \\ ${ }^{4}$ National Radio Astronomy Observatory, 520 Edgemont Road, Charlottesville, VA 22903, USA \\ ${ }^{5}$ Department of Physics and Astronomy, Rice University, 6100 South Main, Houston, TX 77521-1892, USA \\ Received 2014 August 13; accepted 2014 October 29; published 2015 January 8
}

\begin{abstract}
We present the Atacama Large Millimeter/submillimeter Array (ALMA) continuum observations at a wavelength of $1.25 \mathrm{~mm}$ of the debris disk surrounding the $\sim 100 \mathrm{Myr}$ old solar analog HD 107146. The continuum emission extends from about 30 to $150 \mathrm{AU}$ from the central star with a decrease in the surface brightness at intermediate radii. We analyze the ALMA interferometric visibilities using debris disk models with radial profiles for the dust surface density parameterized as (1) a single power law, (2) a single power law with a gap, and (3) a double power law. We find that models with a gap of radial width $\sim 8 \mathrm{AU}$ at a distance of $\sim 80 \mathrm{AU}$ from the central star, as well as double power-law models with a dip in the dust surface density at $\sim 70 \mathrm{AU}$ provide significantly better fits to the ALMA data than single power-law models. We discuss possible scenarios for the origin of the HD 107146 debris disk using models of planetesimal belts in which the formation of Pluto-sized objects trigger disruptive collisions of large bodies, as well as models that consider the interaction of a planetary system with a planetesimal belt and spatial variation of the dust opacity across the disk. If future observations with higher angular resolution and sensitivity confirm the fully depleted gap structure discussed here, a planet with a mass of approximately a few Earth masses in a nearly circular orbit at $\sim 80 \mathrm{AU}$ from the central star would be a possible explanation for the presence of the gap.
\end{abstract}

Key words: circumstellar matter - planets and satellites: formation - stars: individual (HD 107146) submillimeter: stars

\section{INTRODUCTION}

Observations of debris disks made of cold dust around mainsequence stars can provide crucial information about the planet formation process (e.g., Zuckerman 2001; Matthews et al. 2014). The dust grains observed in these systems arise from the material left over from the formation of planets, being continuously replenished by collisions of larger bodies, such as comets and asteroids (see, e.g., Wyatt 2008). Mapping the structure of debris disks is important since the spatial distribution of dust is potentially a powerful diagnostic of the evolution of planetary systems as planets interact gravitationally to sculpt the disk.

Whereas optical and infrared observations of disks trace micron-sized grains that can be either pushed away by the pressure of the radiation from the central star or migrate inward because of Poynting-Robertson drag, millimeter grains traced by observations in the (sub-)millimeter are relatively insensitive to these mechanisms (Burns et al. 1979). Thus the location of the millimeter-sized dust will trace where the large parent bodies, i.e., comets and asteroids, are located in the circumstellar disk, and the spatial variations in the dust density will reflect the dynamical history of the disk and planetary system (Wyatt 2006).

At a Hipparcos-measured distance of $27.5 \pm 0.4$ pc (van Leeuwen 2007) HD 107146 has the same spectral type as the Sun (G2V) and an age of $\sim 80-200 \mathrm{Myr}$ (Moor et al. 2006). HD 107146 was discovered to posses a debris disk from analysis of IRAS data (Silverstone 2000). An analysis of Spitzer spectroscopic and photometric data revealed the presence of two components, a warm one with dust temperature of $\approx 120 \mathrm{~K}$ located $\sim 5-15$ AU from the central star, and a colder component with temperature of $\approx 50 \mathrm{~K}$ at larger radii (Morales et al. 2011). The disk was resolved in scattered light by the Hubble Space Telescope (HST; Ardila et al. 2004; Ertel et al. 2011; Schneider et al. 2014), and in the (sub-)millimeter with the James Clerk Maxwell Telescope (Williams et al. 2004). The scattered light images reveal an unusually broad (FWHM $\approx 90 \mathrm{AU}$ ) ring centered around $\approx 130$ AU. Thus the HD 107146 debris disk is a larger version of the Kuiper belt.

The nearly face-on orientation of the disk and its high brightness at far-infrared wavelengths, a factor of $\sim 4-5$ higher than any other known debris disk around nearby G-type stars (Moor et al. 2006), makes it an ideal case to image a debris disk around a young solar analog at high sensitivity and angular resolution in the sub-millimeter. Corder et al. (2009) and Hughes et al. (2011) used the Combined Array for Research in Millimeter-wave Astronomy (CARMA), and the Submillimeter Array (SMA), respectively, to constrain the density structure of mm-grains in the disk. While Corder et al. (2009) suggest that the disk may be clumpy at millimeter wavelengths, Hughes et al. (2011) imaged the disk with higher signal-to-noise ratio and found that the disk is consistent with an azimuthally symmetric ring. However, these observations were still limited in terms of sensitivity and angular resolution: at the distance of HD 107146 their angular resolution translates into a spatial resolution of about $70 \mathrm{AU}$, so that the ring width could be resolved only marginally.

We present new observations of the HD 107146 debris disk obtained at a wavelength of $1.25 \mathrm{~mm}$ with the Atacama Large Millimeter/submillimeter Array (ALMA) in Cycle 0. Compared to previous submillimeter observations, the ALMA data are more than an order of magnitude more sensitive and have two to three times better angular resolution, and therefore provide 
Table 1

ALMA Observations

\begin{tabular}{lcccccc}
\hline \hline UT Date & Number & Baseline Range & pwv & \multicolumn{3}{c}{ Calibrators } \\
\cline { 5 - 7 } & Antennas & $(\mathrm{m})$ & $(\mathrm{mm})$ & Flux & Passband & Gain \\
\hline 2012 Jan 11 & 17 & $19-269$ & 2.29 & Mars & $3 \mathrm{C} 273$ & J1224+213 \\
2012 Jan 27 & 16 & $19-269$ & 3.02 & Mars & $3 \mathrm{C} 273$ & $\mathrm{~J} 1224+213$ \\
2012 Jan 27 & 16 & $19-269$ & 2.86 & Mars & $3 \mathrm{C} 273$ & $\mathrm{~J} 1224+213$ \\
2012 Dec 16 & 23 & $15-382$ & 1.13 & Titan & $3 \mathrm{C} 273$ & $\mathrm{~J} 1224+213$ \\
2013 Jan 1 & 24 & $15-402$ & 2.82 & Titan & 3C273 & J1224+213 \\
\hline
\end{tabular}

Notes. ${ }^{\text {a }}$ Data have high phase noise and was not included in the imaging and model fitting.

better constraints on the spatial distribution of millimeter-sized grains in the debris disk.

\section{OBSERVATIONS AND DATA REDUCTION}

ALMA observations of the $1.25 \mathrm{~mm}$ dust continuum and $\mathrm{CO}$ $J=2-1$ toward HD 107146 were obtained in Cycle 0 in five observing blocks. Table 1 summarizes the observations, including the date, the number of $12 \mathrm{~m}$ antennas used, the minimum and maximum projected baselines, the median precipitable water vapor at zenith, the primary flux calibrator, the passband calibrator, and the gain calibrator for each observing block. The data in the second observing block obtained on 2012 January 27 had significantly higher phase noise than the other four observing blocks and is not considered further in the analysis.

Dual-polarization observations were obtained in four bands centered on frequencies of $230.5,232.5,246.5$, and $248.5 \mathrm{GHz}$ for a mean frequency of $239.5 \mathrm{GHz}(\lambda=1.25 \mathrm{~mm})$. The first spectral window encompasses the $J=2-1$ rotational transition of $\mathrm{CO}$ with a rest frequency of $230.538 \mathrm{GHz}$. Each spectral window was configured to provide a bandwidth of $1.875 \mathrm{GHz}$ per polarization with a channel spacing of $0.488 \mathrm{MHz}\left(0.63 \mathrm{~km} \mathrm{~s}^{-1}\right.$ for $\left.\mathrm{CO} J=2-1\right)$. The spectral resolution is twice the channel spacing since the data are Hanning smoothed.

The ALMA data were calibrated by NRAO staff using the CASA software package version 4.1 (McMullin et al. 2007). The frequency-dependent bandpass was calibrated by observing 3C273. Simultaneous observations of the $183 \mathrm{GHz}$ water line with the water vapor radiometers were used to reduce atmospheric phase noise before using $\mathrm{J} 1224+213$ for complex gain calibration. Flux calibration was established by observing either Mars or Titan, and adopting the Butler-JPL-Horizon 2012 models, resulting in an accuracy of $10 \%$.

Since the dates of the HD 107146 observations were not known a priori, the phase center for all observations were set to $(\alpha, \delta)=(12: 19: 06.358,16: 32: 52.091) \mathrm{J} 2000$, which is approximately the position of HD 107146 in 2012 January after correcting for a proper motion of $(\Delta \alpha, \Delta \delta)=(-0.174$, -0.149) $\operatorname{arcsec} \mathrm{yr}^{-1}$ (van Leeuwen 2007). To account for proper motion of the course of the year of observations (see Table 1), the visibility phases were adjusted for the change in position relative to data in the first observing block. The offset of the stellar position relative to the phase center is $(\Delta \alpha, \Delta \delta)=(-0.02 \pm 0.01,-0.02 \pm 0.02)$ arcsec, where the uncertainties include uncertainties in the stellar position and proper motion.

The uncertainties in the visibilities contained in the delivered ALMA data reflect the relative system temperatures in the receivers on the antennas but not other factors (integration time, channel width, and correlator efficiency) that are needed for

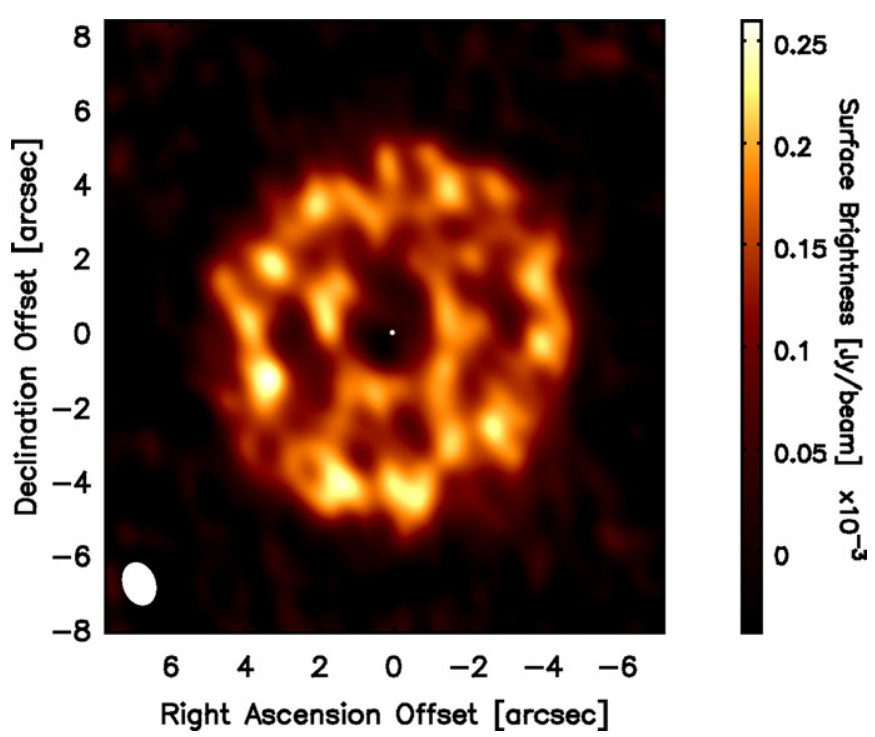

Figure 1. ALMA $1.25 \mathrm{~mm}$ continuum map of HD 107146 where imaging was performed with natural weighting, i.e., Briggs robust parameter of 2 . Offsets in right ascension and declination are relative to the phase center of the observations. The white ellipse in the lower left corner represents the synthesized beam with FWHM size of $1^{\prime \prime} .15 \times 00^{\prime \prime} .84$ and position angle of $19.8 \mathrm{deg}$. The small white dot toward the center of the map indicates the location of the star (see Section 2).

an absolute measurement uncertainty (S. Schnee 2014, private communication). We empirically derived the factor needed to scale from the relative to absolute uncertainties. For each observing block and each spectral window, the visibility data within a window were channel-averaged and gridded in $(u, v)$ space. The dispersion of the visibility measurements within each cell were then computed. The median ratio between the observed dispersion within a cell and the uncertainties reported in the ALMA data was computed using cells containing $\geqslant 10$ visibilities. The uncertainties in the visibilities for that window and observing block were scaled by that ratio.

\section{CONTINUUM AND SPECTRAL LINE IMAGES}

\subsection{Continuum Images}

The CASA task clean was used to Fourier invert the complex visibilities to create an image of the dust emission and to perform multi-frequency synthesis deconvolution. Figure 1 presents the ALMA map of the $\lambda 1.25 \mathrm{~mm}$ continuum emission from HD 107146 obtained with natural weighting using a Briggs robust parameter of 2 . This weighting scheme gives a synthesized beam of $1^{\prime \prime} .15 \times 0.0^{\prime \prime} 84$, corresponding to a spatial resolution of about $32 \times 23 \mathrm{AU}$ at the distance of HD 107146 . Figure 2 shows the same ALMA data but weighted with a Gaussian $u v$-taper with an on-sky FWHM of 2 arcsec. This suppresses the weight of the longer baselines, with the aim of highlighting the more diffuse emission from the debris disk. The angular resolution of the map of $2^{\prime \prime} .52 \times 2^{\prime \prime} .25$ is about twice as large as the map obtained with natural weighting.

The integrated flux density from the disk is $12.5 \pm 1.3 \mathrm{mJy}$ at $1.25 \mathrm{~mm}$. This was obtained by integrating the surface brightness over the area showing emission at $\gtrsim 2 \sigma$ above the background level in the outer taper weighted map. The uncertainty reflects the $10 \%$ of uncertainty on the absolute flux scale, whereas the $1 \sigma$ noise levels on the natural and outer taper weighted maps are 0.030 and $0.057 \mathrm{mJy}^{\text {beam }}{ }^{-1}$, respectively. This flux density is consistent at $\approx 1 \sigma$ with the measurement 


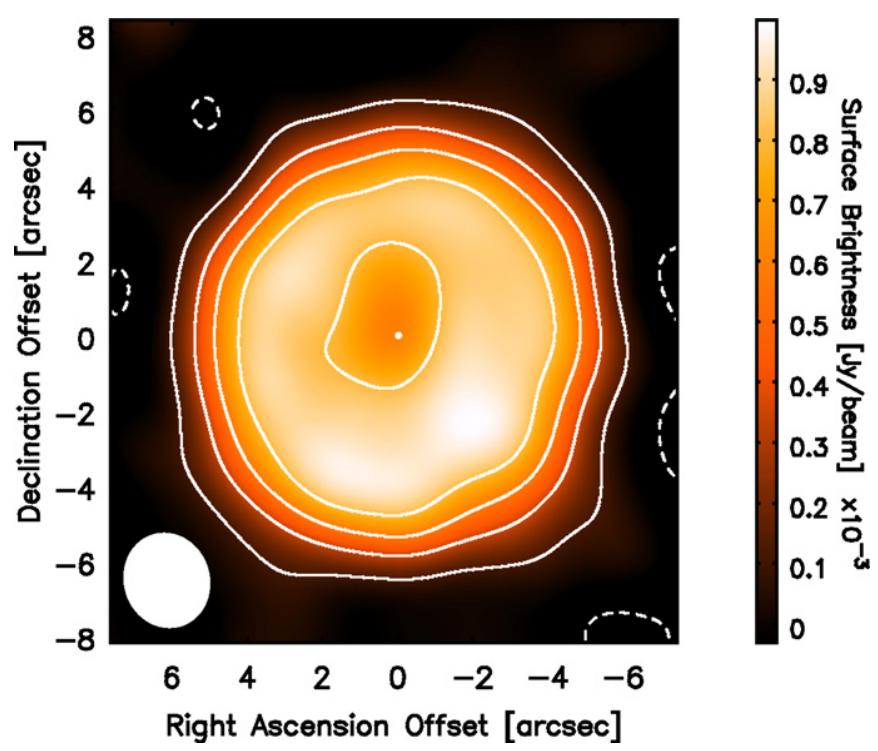

Figure 2. Same as in Figure 1, except the visibility data have been tapered by a Gaussian with an on-sky FWHM of 2 arcsec. White contours are drawn at $-2 \sigma$ (dashed line), $2 \sigma, 6 \sigma, 10 \sigma, 14 \sigma$ (solid), where $1 \sigma=57 \mu \mathrm{Jy}_{\text {beam }}{ }^{-1}$. The white ellipse in the lower left corner represents the synthesized beam with FWHM size of $2^{\prime \prime} .52 \times 2^{\prime \prime} .25$ and position angle of $17.0 \mathrm{deg}$. The small white dot toward the center of the map indicates the location of the star.

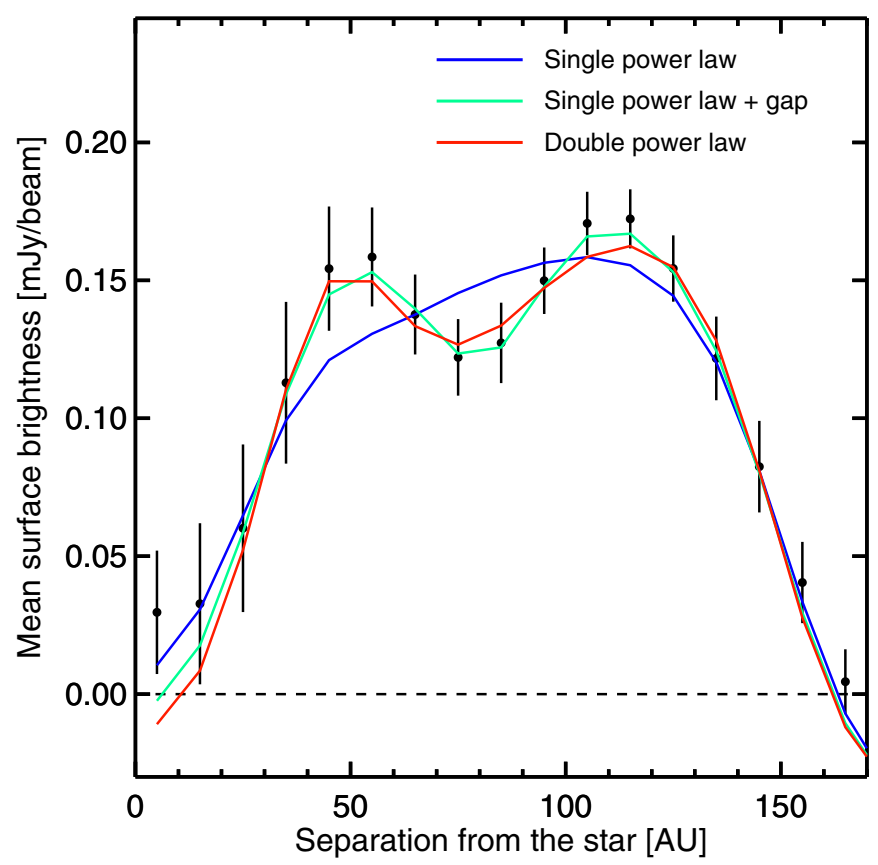

Figure 3. Mean surface brightness of the HD 107146 debris disk as a function of the physical separation from the central star. Black data points show the mean surface brightness derived from the natural-weight ALMA map using elliptical photometry. The position angle and aspect ratio of the elliptical regions used for the photometry reflect the P. A. and inclination of the best-fit single power-law model with gap, respectively (Table 2). The error bars reflect the uncertainty of the mean within each elliptical region. Colored continuous lines show the mean surface brightness radial profiles from the best-fit models for the three classes of models as labeled in the figure.

of $10.4 \pm 1.4 \mathrm{mJy}$ by Corder et al. (2009) using CARMA observations at a slightly longer effective wavelength of about $1.32 \mathrm{~mm}$.

Figure 3 shows the radial averaged surface brightness profile obtained from the ALMA image with natural weighting. Consistent with previous results from CARMA (Corder et al.
2009) and the SMA (Hughes et al. 2011), the dust emission from HD 107146 extends over an angular diameter of $\sim 11^{\prime \prime}$ with a decrease in the amount of emission toward the star. While the surface brightness of the inner and outer disk are similar, the ALMA observations reveal a decrease in the surface brightness at intermediate radii. These characteristics cannot be caused by spatial filtering from the interferometer. The shortest baseline in the ALMA data is $15 \mathrm{~m}$, which corresponds to angular size scales of $17^{\prime \prime}$, a factor of $\sim 1.5$ larger than the disk. Also, the primary beam size of the ALMA 12 m diameter antennas is $24^{\prime \prime}$ at a wavelength of $1.3 \mathrm{~mm}$. Although these observations are likely not fully sensitive to emission from the largest angular scales of the debris disk (see Wilner \& Welch 1994), the fact that the recovered flux is consistent with that measured by Corder et al. (2009) with baselines shorter by a factor of $\approx 2.5$ indicates that the loss of flux in our data is only marginal. Nonetheless, to avoid possible biases due to an incomplete $(u, v)$ coverage of the observations, our analysis of the disk structure was performed on the measured visibilities (see Section 4).

$$
\text { 3.2. } C O J=2-1
$$

We imaged the CO $J=2-1$ line using different weighting schemes, each providing different synthesized beams and sensitivities to surface brightness. A range of velocities of $\pm 10 \mathrm{~km} \mathrm{~s}^{-1}$ from the known radial velocity of the $\operatorname{star}\left(2.6 \mathrm{~km} \mathrm{~s}^{-1}\right.$; Valenti \& Fischer 2005) was examined. No detection was obtained in any of these attempts by integrating over the disk size as seen in dust continuum. The tightest upper limit to surface brightness, and therefore to column density in $\mathrm{CO}$, that could be derived from our data is about $10 \mathrm{mK}$ (at $3 \sigma$ ) with an approximate angular resolution of $6^{\prime \prime}$. A discussion on the upper limit on the mass in $\mathrm{CO}$ derived by this non-detection is presented in Section 7.

\section{ANALYSIS OF THE OBSERVED INTERFEROMETRIC VISIBILITIES}

We now fit the ALMA observations with disk models to account for the observed features in the images: the broad annulus and the decrease in dust emission at intermediate disk radii. We describe in Section 4.1 the general model that will be fitted to the data, and describe the fitting procedure in Section 4.2. We then use the techniques to fit a single power law to the ALMA data (Section 5.1) and explore if more sophisticated surface density profiles are required by the data (Sections 5.2 and 5.3).

\subsection{Debris Disk Model}

Our models consider a debris disk as an axisymmetric, geometrically thin and optically thin layer of solids. At any radial location in the disk $r$, the temperature $T(a, r)$ of a grain with size $a$ is derived from the balance between the energy absorbed by the grain and the thermal energy emitted by the grain itself:

$$
\begin{aligned}
\pi a^{2} & \int_{0}^{\infty} \frac{L_{v}^{\star}}{4 \pi r^{2}} Q_{v}^{\mathrm{abs}}(a) d v \\
& =4 \pi a^{2} \int_{0}^{\infty} Q_{v}^{\mathrm{abs}}(a) \pi B_{v}[T(a ; r)] d \nu .
\end{aligned}
$$

In Equation (1), $L_{v}^{\star}$ is the stellar monochromatic luminosity, $Q_{v}^{\text {abs }}(a)$ is the frequency dependent absorption efficiency of a grain with size $a$, and $B_{v}[T(a ; r)]$ is the Planck function at 
the temperature of the grain. After defining the stellar and dust properties, through $L_{v}^{\star}$ and $Q_{v}^{\mathrm{abs}}(a)$, respectively, Equation (1) can be solved numerically to derive the temperature $T(a ; r)$. In the case of HD 107146, $L_{v}^{\star}$ was derived from a black body with effective temperature $T=5841 \mathrm{~K}$ and bolometric luminosity $\log \left(L_{\text {bol }} / L_{\odot}\right)=0.04$ (Carpenter et al. 2008; Hillenbrand et al. 2008). The absorption coefficients $Q_{v}^{\mathrm{abs}}(a)$ were extracted from the dust models of Ricci et al. (2010a), which consider spherical porous solids made of a mixture of silicates, carbonaceous materials, water ice (optical constants from, Weingartner \& Draine 2001; Zubko et al. 1996; Warren 1984, respectively,) and vacuum. These are based on the Pollack et al. (1994) models for dust in protoplanetary disks, and derived by an analysis of astronomical data and theory, as well as the composition of primitive bodies in the solar system.

An implicit assumption used to derive Equation (1) is that the whole disk is radially optically thin to the stellar radiation, so that stellar photons can reach any region in the disk without significant absorption. This assumption was verified a posteriori on the disk models that can reproduce the ALMA data for HD 107146.

The radial profile of the surface brightness of the disk as viewed as face-on is given by

$$
I_{v}(r)=\frac{\Sigma(r) \int_{a_{\min }}^{a_{\max }} B_{v}[T(a ; r)] n(a) a^{3} \kappa_{v}^{1}(a) d a}{\int_{a_{\min }}^{a_{\max }} n(a) a^{3} d a},
$$

where $\Sigma(r)$ is the radial surface density of dust, $n(a)$ is the grain size distribution, and $\kappa_{v}^{1}(a)$ is the single-grain dust opacity coefficient that is related to the absorption efficiency through $(3 / 4 a \rho) Q_{v}^{\text {abs }}(a)$, with $\rho=1.2 \mathrm{~g} \mathrm{~cm}^{-3}$ being the mean solid density for the Ricci et al. (2010a) dust model considered here (see Miyake \& Nakagawa 1993).

Sub-millimeter continuum observations can be used to constrain the size distribution of dust particles in debris disks. The grain size distribution is commonly approximated as a powerlaw function $d N=n(a) d a, n(a) \propto a^{-q}$ for grains between a minimum and maximum grain size $a_{\min }$ and $a_{\max }$, respectively. For grain size distributions that follow a power law with $3<q<4$ over a broad enough interval around the observation wavelength, i.e., $a_{\min } \ll \lambda \ll a_{\max }$, Draine (2006) derived a relation between $q$, the spectral index $\beta$ of the dust opacity $\left(\kappa_{v} \propto v^{\beta}\right)$, and the spectral index $\beta_{s}$ of small grains $(a \ll \lambda)$, $\beta_{s}=1.8 \pm 0.2$ for interstellar grains. The assumption that the smallest and largest grains in the dust population are much smaller and larger than $\lambda \approx 1 \mathrm{~mm}$, respectively, is in line with the interpretation of debris dust produced by a collisional cascade of large planetesimals getting ground all the way down to $\sim \mu \mathrm{m}$ sized grains. Also, physical models of collisional cascades of planetesimals predict $q$-values between about 3 and 4 (Dohnanyi 1969; Pan \& Schlichting 2012; Gaspar et al. 2012), so that we can use the Draine (2006) equation to derive an estimate for $q$ after inferring $\beta$ (see Ricci et al. 2012).

The spectral index $\beta$ of the dust opacity can be constrained by measuring the spectral index $\alpha$ of the millimeter spectral energy distribution $\left(F_{v} \propto v^{\alpha}\right)$. By combining the flux densities measured for the HD 107146 debris disk at $0.88 \mathrm{~mm}, 1.25 \mathrm{~mm}$, $1.32 \mathrm{~mm}$, and $3.0 \mathrm{~mm}$ by Hughes et al. (2011), this work, Corder et al. (2009) and Carpenter et al. (2005), respectively, we measured a millimeter spectral index $\alpha=2.42 \pm 0.16$.

In the case of HD 107146, the results of our modeling presented in Section 4 show that the dust emission at these wavelengths is optically thin and in the Rayleigh-Jeans regime and therefore the spectral index of the dust opacity is given by $\beta=\alpha-2$ (see, e.g., Ricci et al. 2010a). We then obtained $q=3.25 \pm 0.09$ from the Draine (2006) relation.

The integrals in Equation (2) are formally computed over all sizes of solids in the disk. A good approximation for the minimum grain size $a_{\mathrm{min}}$ is the blow-out grain size $a_{\mathrm{blow}}$, as grains smaller than $a_{\text {blow }}$ are blown out of the system by the stellar radiation field as soon as they are created. We estimated $a_{\text {blow }} \approx 1.7 \mu \mathrm{m}$ using Equation (2) from Roccatagliata et al. (2009) with an albedo of $\approx 0.5$ from our dust model, and a stellar mass of $1.0 M_{\odot}$ for HD 107146. With the equation used by Ricarte et al. (2013), which is valid for a silicate dust particle on a circular orbit around the central star, we would get $a_{\text {blow }} \approx 2.7 \mu \mathrm{m}$. However, our analysis is insensitive to this variation for $a_{\text {blow }}$ since the emission at the ALMA wavelength of $1.25 \mathrm{~mm}$ is dominated by grains much larger than few $\mu \mathrm{m}$.

The largest solids in a debris disk are kilometer-sized planetesimals or even planet-sized objects if these are present in the system. However, for practical reasons, the integrals in Equation (2) can be computed up to just a few centimeters as the emission from these and larger grains is negligible at the wavelength of our observations. For this reason we adopted $a_{\max }=2 \mathrm{~cm}$.

After adopting a parameterization for the dust surface density $\Sigma(r)$ (see Section 5), Equations (1) and (2) define the debris disk model. The free parameters of our models are the parameters used to describe $\Sigma(r)$, the disk inclination $i$ defined as the angle between the disk axis and line-of-sight direction $\left(0^{\circ}\right.$ for face-on geometry), the disk position angle (P.A.) defined as the angle east of north to the disk major axis, and the offsets $\Delta \alpha$ and $\Delta \delta$ of the disk center relative to the phase center of the ALMA observations.

\subsection{Models-Data Comparison}

The models were fitted to the complex visibilities rather than the images to avoid non-linear effects of the image deconvolution, correlated noise between image pixels, as well as possible filtering out of the disk large scale emission. For a given set of disk model parameters, an image of the dust emission was generated at the mean frequency $(239.5 \mathrm{GHz})$ of the observations. The image was then multiplied by the primary beam response of a $12 \mathrm{~m}$ ALMA antenna assuming a Gaussian-shaped beam with an FWHM of 24".3. The model image has a size of $2048 \times 2048$ pixel with 0.015 pixels. The model visibilities were obtained through a Fourier transform of the image and sampling the model using the same $(u, v)$ data points in the ALMA observations. The flux density of the sampled visibilities were varied with the frequency of the spectral bands as $\nu^{2+\beta}$, where $\beta=0.4$. The value of $\chi^{2}$ for the model parameters can then be computed.

The best-fit model parameters and parameter uncertainties were found using emcee, an MIT licensed pure-Python implementation of the Goodman \& Weare (2010) Affine Invariant Markov Chain Monte Carlo (MCMC) Ensemble sampler ${ }^{5}$ (Foreman-Mackey et al. 2013). Relative to the traditional methods of $\chi^{2}$-minimization over a fixed multi-dimensional grid, the emcee algorithm has the advantage of focusing on the regions of the parameter space around the $\chi^{2}$ absolute minimum. The models which were accepted by the MCMC Ensemble sampler were used to probe the probability function for each model parameter obtained through marginalization, i.e., by integrating the posterior distribution over all the parameters except the one

\footnotetext{
For more information on emcee see http://dan.iel.fm/emcee/current/.
} 
Table 2

Constraints on the Model Parameters from the Analysis of the ALMA Visibilities for the Three Classes of Models Discussed in Section 5

\begin{tabular}{lccc}
\hline \hline Parameter & Single Power Law & Single Power Law with Gap & Double Power Law \\
\hline$R_{\text {in }}[\mathrm{AU}]$ & $25.2_{-1.8}^{+2.7}$ & $30.3_{-0.9}^{+2.4}$ & $35.8_{-1.8}^{+1.3}$ \\
$R_{\text {gap }}[\mathrm{AU}]$ & $\ldots$ & $80.9_{-2.6}^{+1.8}$ & $\ldots$ \\
$\Delta R_{\text {gap }}[\mathrm{AU}]$ & $\ldots$ & $9.0_{-1.5}^{+1.0}$ & $\ldots$ \\
$R_{\text {out }}[\mathrm{AU}]$ & $152.0_{-1.8}^{+1.0}$ & $150.4_{-1.1}^{+1.7}$ & $148.3_{-0.7}^{+2.1}$ \\
$\log \left[\Sigma_{0} /\left(\mathrm{g} \mathrm{cm}^{-2}\right)\right]$ & $-5.58_{-0.09}^{+0.13}$ & $-5.23_{-0.06}^{+0.20}$ & $-2.33_{-1.21}^{+0.21}$ \\
$p$ & $0.74_{-0.05}^{+0.06}$ & $0.59_{-0.09}^{+0.04}$ & $\ldots$ \\
$p_{1}$ & $\ldots$ & $\ldots$ & $-1.09_{-0.11}^{+0.74}$ \\
$p_{2}$ & $\ldots$ & $\ldots$ & $1.37_{-0.20}^{+0.08}$ \\
$R_{\text {break }}[\mathrm{AU}]$ & $\ldots$ & $\ldots$ & $68.5_{-2.6}^{+6.2}$ \\
$i$ [deg] & $20.6 \pm 1.9$ & $20.7_{-2.3}^{+2.2}$ & $21.0_{-1.9}^{+2.0}$ \\
P.A. $[$ deg] & $144.7_{-4.2}^{+7.1}$ & $141.1_{-3.3}^{+7.9}$ & $143.0_{-3.6}^{+7.4}$ \\
$\Delta \alpha$ arcsec] & $0.048_{-0.027}^{+0.039}$ & $0.029_{-0.039}^{+0.021}$ & $0.046_{-0.023}^{+0.035}$ \\
$\Delta \delta[\operatorname{arcsec}]$ & $-0.123_{-0.031}^{+0.048}$ & $-0.081_{-0.020}^{+0.046}$ & $-0.054_{-0.037}^{+0.030}$ \\
\hline
\end{tabular}

of interest. The best-fit values and (asymmetric) uncertainties presented for each parameter in this work reflect the mode of the marginalized probability distribution and the $68.3 \%$ confidence level, respectively.

For each surface density parameterization, emcee was run with several hundreds walkers (see Foreman-Mackey et al. 2013) for several hundreds iterations. We also ran several trials by either varying or not varying the starting positions of the walkers to confirm that the final constraints on the model parameters are not affected by the randomly Gaussian distributed walkers and/or changes in the starting values of the parameters.

\section{MODEL RESULTS}

In this section we describe the results of the modeling analysis. Our philosophy was to choose simple parameterizations of the dust surface density that capture the key features seen in the ALMA images shown in Figures 1 and 2. We begin by fitting a single power-law surface density to the data to describe the broad annulus, and then show that more intricate models that allow for a decline in the surface density at a radius of $\approx 70-80 \mathrm{AU}$ provide statistically better fits to the data.

\subsection{Single Power-law Models}

The simplest parameterization we considered to describe $\Sigma(r)$ is a power law truncated at an inner and outer radius, $R_{\text {in }}$ and $R_{\text {out }}$, respectively,

$$
\Sigma(r)= \begin{cases}\Sigma_{0}\left(\frac{r}{1 \mathrm{AU}}\right)^{p} & \text { where } R_{\mathrm{in}}<r<R_{\text {out }} \\ 0 & \text { elsewhere, }\end{cases}
$$

where $r$ is the distance in the disk from the central star, $\Sigma_{0}$ is the surface density normalized at $1 \mathrm{AU}^{6}{ }^{6}$ and $p$ is the slope of the radial power law. The model thus contains eight free parameters: $\left\{\Sigma_{0}, p, R_{\text {in }}, R_{\text {out }}, i\right.$, P.A., $\left.\Delta \alpha, \Delta \delta\right\}$.

Table 2 reports the constraints on the model parameters derived by our analysis (uncertainties are at $1 \sigma$ ). The top row in Figure 4 shows the probability distribution for $R_{\text {in }}, R_{\text {out }}$, $p$, as derived using the emcee algorithm (Section 4.2). With these models, the disk extends from about 25 to $152 \mathrm{AU}$. These estimates for inner and outer radii are both smaller than the

\footnotetext{
6 With this definition $\Sigma_{0}>0$ even when $R_{\text {in }}>1$ AU.
}

values of 50 and $170 \mathrm{AU}$, respectively, derived by Hughes et al. (2011) from the combined analysis of the spectral energy distribution and SMA interferometric data at $0.88 \mathrm{~mm}$ using single power-law models, but are still within the uncertainties, reported as larger than $10 \mathrm{AU}$ in their analysis.

The $p$ value of $0.74_{-0.05}^{+0.06}$ indicates a surface density that is increasing with radius, i.e., $p>0$. This is in line with the estimate of $\Sigma(r) \propto r^{0.3 \pm 0.3}$ found by Hughes et al. (2011), although their large uncertainty did not allow them to rule out a flat or decreasing radial profile with their data. This result can be understood by noticing that at our wavelength of $1.25 \mathrm{~mm}$ $I_{\nu} \propto B_{v}[T(r)] \times \Sigma(r) \sim T(r) \times \Sigma(r) \sim r^{-0.5} \times \Sigma(r)$, and that as seen in Figure 1, the surface brightness toward the inner and outer edges of the disk is roughly the same. The offsets $\Delta \alpha$ and $\Delta \delta$ of the disk center relative to the phase center are consistent, within $\approx 2 \sigma$, with the offsets of the star (Section 2). This is true also for the other classes of models discussed in the next sections.

The value of the minimum of the $\chi^{2}$ function is 2519918.4, which corresponds to a reduced $\tilde{\chi}^{2}$ of 1.028 . The image on the top left corner in Figure 5 contains the synthetic map of the best-fit model, which shows how that model would have looked like if it was observed under the same conditions (i.e., same $(u, v)$ coverage, sensitivity) as our actual ALMA observations, with imaging performed using natural weighting. The contours in the right top corner map in the same figure show the residuals of the data-model subtraction. Although for the majority of the disk surface the residuals are below the $2 \sigma$ level, some peaks and dips at $\sim 2 \sigma-3 \sigma$ are seen. In particular, nearly all these peaks (shown as blue continuous lines in the map) are located close to either the inner or the outer edge of the disk where the disk surface brightness is high, whereas the dips (blue dashed lines) are more toward the middle of the ring where the disk surface brightness is lower (see color map in the top right panel in Figure 5). This behavior is also evident in the radial profile of the disk surface brightness shown in Figure 3. This suggests that a surface density radial profile that can account for a depletion region at intermediate radii between $R_{\text {in }}$ and $R_{\text {out }}$ will likely better reproduce the ALMA data than a single continuous power law. In order to test this, in the next two subsections we present two simple extensions to the single power-law models.

Figure 6 shows the comparison between visibility data measured by ALMA and binned over deprojected baseline lengths 

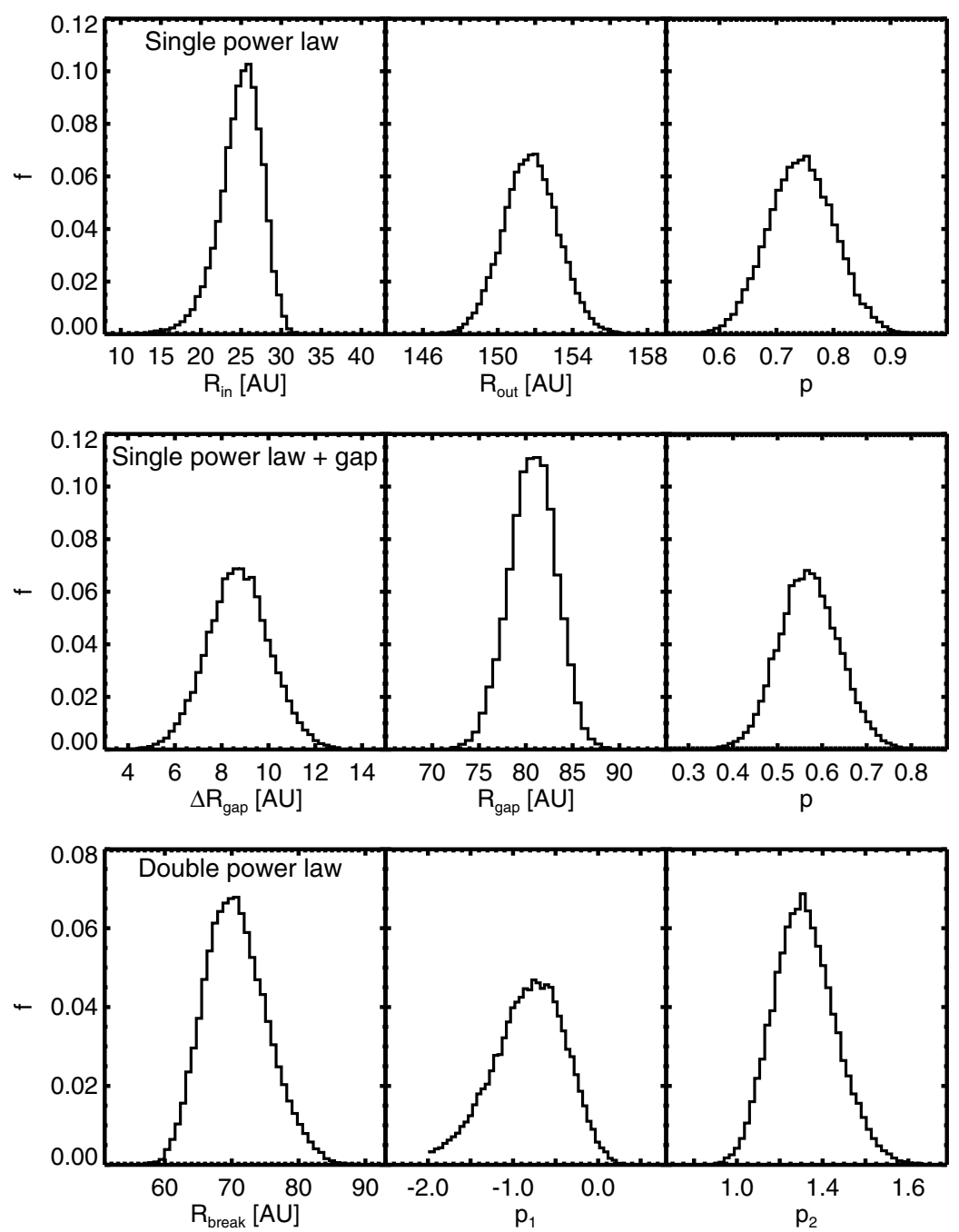

Figure 4. Normalized probability distributions for some of the model parameters as obtained from the emcee fitting process. The three rows present the normalized distribution of parameters for single power-law (top row), single power-law with gap (middle), double power-law models (bottom), respectively. The model parameters are those defined in Sections 5.1-5.3.

and the prediction of the single power-law model (blue line). The model shown here has parameters values corresponding to the peak of the probability distributions marginalized over each model parameter as probed by the emcee algorithm (Section 4.2). The small plot on the top of the figure highlights a region where the single power-law model does not reproduce well the real part of the visibility data. This occurs at deprojected baseline lengths of $\approx 75-130 \mathrm{~m}$, or $\approx 60-110 \mathrm{k} \lambda$. These baseline lengths correspond to angular scales of $\approx 2-3$ arcsec on the sky that is also the approximate range of angular distances from the central star of the decrease in surface brightness in the disk.

\subsection{Single Power Law with Gap}

A simple extension to the single power-law models that can account for a radial depletion of dust is provided by the following surface density functions:

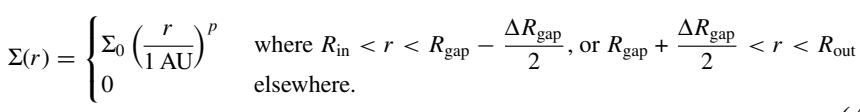

Equation (4) represents a single power law truncated at $R_{\text {in }}$ and $R_{\text {out }}$ as in Equation (3) with the addition of an axisymmetric gap with $\Sigma(r)=0$ centered at $R_{\text {gap }}$ and width $\Delta R_{\text {gap }}$. This models has the same 8 parameters as the single power-law models
(Section 5.1) plus two additional parameters that define the gap, namely $R_{\text {gap }}$ and $\Delta R_{\text {gap }}$, for a total of 10 model parameters.

The constraints to the model parameters are reported in the third column of Table 2. The probability distributions estimated for $\Delta R_{\text {gap }}, R_{\text {gap }}$ and $p$ are shown in the middle row in Figure 4. The best-fit model presents a gap at a distance of about $80.9_{-2.6}^{+1.8} \mathrm{AU}$ (at the center of the gap) and with a radial width $\Delta R_{\text {gap }}=9.0_{-1.5}^{+1.0} \mathrm{AU}$. The slope $p$ of the power law of the surface density, i.e., $0.59_{-0.09}^{+0.04}$, is only slightly lower but still compatible at $2 \sigma$ with the positive value found in the previous subsections.

The best-fit model has a $\chi^{2}$ value of 2519875.4 , which is lower by $\Delta \chi^{2}=43.0$ than the case of a single power-law model with no gap. These models are an extension of the single powerlaw models presented in Section 5.1, which can be reproduced if $\Delta R_{\text {gap }}=0$ and/or $R_{\text {gap }}=R_{\text {in }}$ or $R_{\text {gap }}=R_{\text {out }}$. In this case we can use the $F$-test to investigate whether this decrease of the $\chi^{2}$ value with models with two extra parameters is statistically significant, i.e., if the best-fit model with a gap can be considered as a statistically better representation of the ALMA data than the single power-law models with no gap. The $F$-test statistics in this case has a value of $8 \times 10^{-10}$, indicating that the models with gap provide a significantly better fit to the ALMA data. A similarly low value of about $3 \times 10^{-9}$ for the relative likelihood 

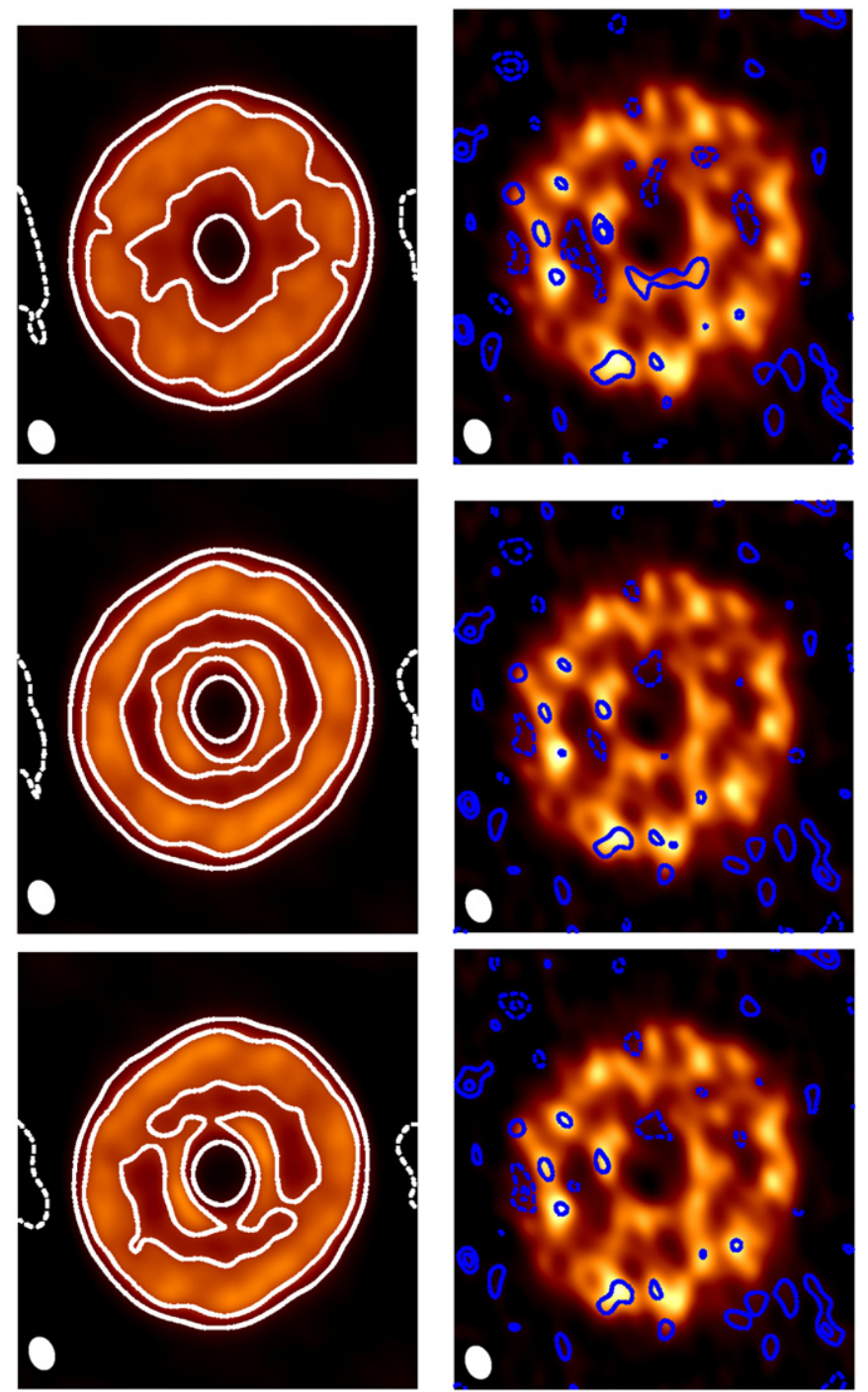

Figure 5. Images of the best-fit model (left column), residuals (right column, contour maps), and ALMA data (right column, color maps) for the HD 107146 debris disk. The three rows show the best-fit model synthetic map and data-best-fit model residual maps for the single power-law models (top row), single power-law models with gap (middle), double power-law models (bottom). For all maps imaging was performed with natural weighting. The size of the images, color scale, and synthesized beam are as in Figure 1. The white contours on the maps in the left column are drawn at $-2 \sigma, 2 \sigma, 5 \sigma$, with $1 \sigma=30 \mu \mathrm{Jy} \mathrm{beam}^{-1}$. The blue contour lines on the residual maps on the right are drawn at $-3 \sigma,-2 \sigma, 2 \sigma, 3 \sigma$, with negative contours drawn as dashed lines.

of these models is derived using the Akaike information criterion (AIC). This is consistent with the fact that in our analysis about $99 \%$ of the models have $\Delta R_{\text {gap }}>5.5 \mathrm{AU}$ (see also Figure 4 ).

The fact that models with a gap better reproduce the ALMA data than single power-law models can be seen also by comparing the map of the data best-fit model residuals between these two classes of models (top two rows in Figure 5). The presence of a gap reproduces the decrease in surface brightness at radii roughly intermediate between the disk inner and outer radii. Also, the surface brightness of the best-fit model with gap is larger than in the no-gap case toward the disk inner and outer radii (see also Figure 3 ). As a consequence, lower absolute values for the residuals are seen in the residual map of the best-fit single power-law model with gap.

The better match between the ALMA data and the models with gap relative to the single power-law models is evident also

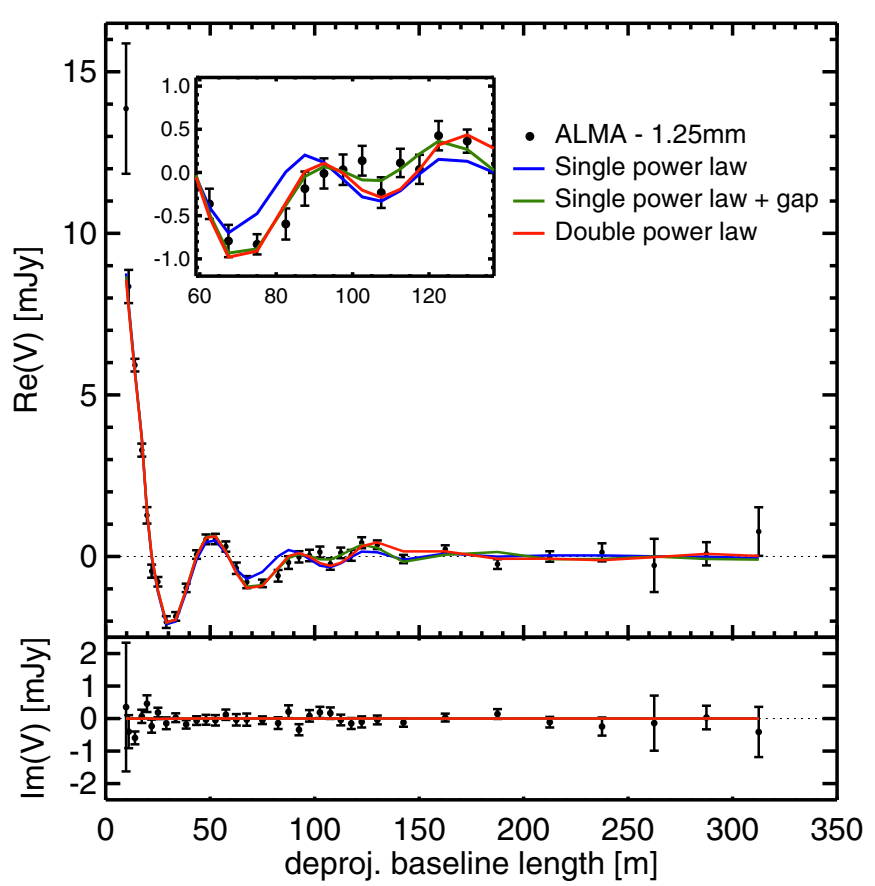

Figure 6. Real and imaginary parts of the visibility function for the HD 107146 debris disk at $1.25 \mathrm{~mm}$ plotted over deprojected baseline length. Black data points represent the ALMA continuum data, color lines are for the models that maximize the likelihood function for the different classes of models as labeled in the figure. The position angle and inclination of these models (see Table 2) were also used to deproject the baseline lengths. The right ascension and declination offsets in Table 2 were used to modify the visibilities so that the phase center corresponds to the center of the disk. The imaginary parts predicted by the models are all zero because all models are axisymmetric by construction.

in Figure 6. The model with gap (green line) fits well the data even in the region with deprojected baseline lengths $\approx 75-135 \mathrm{~m}$, contrary to the single power-law models with no gap.

\subsection{Double Power Law}

The models described in the previous subsection treat the case of a region in the disk that is fully depleted of dust, i.e., a gap. However, the dust depletion may be only partial, its density showing a local decrease but never reaching $\Sigma(r)=0$ for $R_{\text {in }}<r<R_{\text {out }}$. A simple way to account for this possible behavior is by considering a double power-law radial profile for the surface density:

$$
\Sigma(r)= \begin{cases}\Sigma_{0}\left(\frac{r}{1 \mathrm{AU}}\right)^{p_{1}} & \text { where } R_{\text {in }}<r<R_{\text {break }} \\ \Sigma_{\text {break }}\left(\frac{r}{R_{\text {break }}}\right)^{p_{2}} & \text { where } R_{\text {break }}<r<R_{\text {out }} \\ 0 & \text { elsewhere, }\end{cases}
$$

with the condition $\Sigma_{\text {break }}=\Sigma_{0}\left(R_{\text {break }} / 1 \mathrm{AU}\right)^{p_{1}}$ to assure continuity at $r=R_{\text {break }}$, and $R_{\text {in }} \leqslant R_{\text {break }} \leqslant R_{\text {out }}$.

This surface density is described by 6 free parameters, $\left\{\Sigma_{0}, p_{1}, p_{2}, R_{\text {break }}, R_{\text {in }}\right.$, and $\left.R_{\text {out }}\right\}$, for a total of 10 parameters defining this class of models. Also in this case these models represent an extension of the single power-law models discussed in Section 5.1: Equation (5) can "collapse" into Equation (3) if $p_{1}=p_{2}$, and $/$ or $R_{\text {break }}=R_{\text {in }}$ or $R_{\text {break }}=R_{\text {out }}$.

The resulting constraints for the model parameters are reported in the fourth column of Table 2 . The probability distributions estimated for $R_{\text {break }}, p_{1}$ and $p_{2}$ are shown in the bottom row in Figure 4. The best-fit model shows a negative value of $p_{1}$, indicating a surface density that is decreasing with radius out 
to $R_{\text {break }}=68.5_{-2.6}^{+6.2} \mathrm{AU}$, close to the value for the gap radius ( $\left.R_{\text {gap }}=80.9_{-2.6}^{+1.8} \mathrm{AU}\right)$ for models with a gap. Beyond $R_{\text {break }}$ the surface density increases radially with a power law of $p_{2} \approx 1.4$.

The best-fit model has a $\chi^{2}$ value of 2519879.9 . This value is nearly identical (difference of only 4.5) to the best-fit value for the single power law with a gap that has the same number of free parameters. The AIC test returns a relative likelihood of 0.10 between the single power law with a gap and the double power law. We conclude that the difference in the quality of the fit between the two models is not significant. Relative to the single power-law models with no gap, the $\chi^{2}$ is lower by 38.5. The $F$ test statistics has a value of $7 \times 10^{-9}$, and the relative likelihood of these models from the AIC test is $3 \times 10^{-8}$. Therefore, like in the case of a gap, also the addition of a second power law to the surface density radial profile gives a statistically better representation of the ALMA data of HD 107146.

This is supported by the comparison of the residual maps in Figure 5. The residual map for the best-fit double powerlaw model is very similar to the case of a single power-law model with gap and significantly better than the single powerlaw model with no gap. The same occurs for the comparison of the surface brightness (Figure 3) and visibility predictions for these models (Figure 6).

We have also run models where a gap is added to the double power law, and a double power law without the condition of continuity at $R_{\text {break }}$. The general result is that for each functional form considered to analyze the continuum ALMA data of HD 107146, the analysis favors models that show inner and outer radii around $\sim 30$ and $\sim 150$ AU from the star, respectively, a depletion of dust at $\sim 70-80 \mathrm{AU}$, and a dust surface density toward the outer edge of the disk that is comparable or larger than the values found in the inner disk. The ALMA data presented in this paper do not allow us to distinguish between these possible different functional forms, and we decided to show in this paper the models with the lowest numbers of free parameters. This also means that current data do not permit a precise characterization of the radial profile of the dust surface density in the depletion region around $\approx 70-80$ AU from the star. Future ALMA observations with better sensitivity and angular resolution than the ALMA Cycle 0 data presented here are needed for this.

\section{COMPARISON WITH OBSERVATIONS AT SHORTER WAVELENGTHS}

Ardila et al. (2004) and Ertel et al. (2011) observed HD 107146 in scattered light with the HST in the optical and near-infrared, respectively. They found that small, $\mu \mathrm{m}-$ sized grains show a broad radial distribution from $\sim 50-60$ to $\sim 200-250$ AU from the star, with a peak in the derived optical depth at a radius of $\sim 130$ AU. However, given the large subtraction residuals of the point-spread function from the central star, Ardila et al. (2004) cannot rule out the presence of dust within $\sim 60 \mathrm{AU}$ from the star. This general behavior has been confirmed by more recent HST observations with higher image fidelity using the STIS camera (Schneider et al. 2014). Despite the HST observations having better angular resolution than the ALMA ones presented here, the HST maps in scattered light do not highlight any significant depletion region at intermediate disk radii.

The fact that the distribution of dust at short wavelengths appears different from that inferred from the ALMA data at $1.25 \mathrm{~mm}$ is not necessarily surprising. Observations of disks at a given wavelength are mostly sensitive to emission from grains with sizes of the same order of magnitude as the wavelength. Physical mechanisms such as radiation pressure and Poynting-Robertson drag have efficiencies which increase with decreasing grain size. This results in a spatial segregation of grains of different sizes, and therefore different disk morphologies expected at different wavelengths (e.g., Wyatt 2006).

According to the models considered here, half of the flux density at a wavelength of $1.25 \mathrm{~mm}$ is produced by grains with a radius larger than $\sim 1 \mathrm{~mm}$. The ratio of radiation to gravitational forces for these particles is $\sim 0.001$, indicating that radiation pressure has a negligible effect on their dynamics. The opposite is true for smaller $\mu \mathrm{m}$-sized grains, which are instead very sensitive to radiation pressure. The fact that the disk appears more extended in scattered light is likely due to these small grains being pushed outward by radiation pressure. Radiation pressure on $\mu \mathrm{m}$-sized grains may also be the reason why no depletion in the density of dust at intermediate disk radii was seen through high-angular resolution observations at short wavelengths. The same mechanism may explain why these very small particles are found also at larger disk radii than the millimeter-sized grains probed by ALMA, although more sensitive sub-millimeter observations are needed to better characterize those regions with low surface brightness.

Millimeter grains in the HD 107146 disk are insensitive also to Poynting-Robertson drag: their Poynting-Robertson drag timescale is four to five orders of magnitude longer than their collisional timescale, which is of $\sim 10^{4}-10^{5} \mathrm{yr}$ across the disk (Burns et al. 1979). These particles probe the location of the planetesimals that generated them.

By analyzing data from the Infrared Spectrograph and MIPS camera on Spitzer, Morales et al. (2011) suggested the presence of warm dust at distances of 5-15 AU from HD 107146. Given the angular resolution of the ALMA observations, this component would appear as point-like on our images. The fact that we do not detect any emission at the location of the star poses a $3 \sigma$ upper limit of $\approx 2 \times 10^{-4} M_{\oplus}$ for this warm component, after considering a dust temperature of $\approx 120 \mathrm{~K}$ and a population of small grains only, i.e., sizes of $\approx$ few $\mu \mathrm{m}$, as in Morales et al. (2011). This estimate is well consistent with their lower limit of $3 \times 10^{-7} M_{\oplus}$ from the infrared fluxes.

\section{THE NON-DETECTION OF CO}

The non-detection of the ${ }^{12} \mathrm{CO}(J=2-1)$ rotational line can be used to obtain an upper limit on the amount of $\mathrm{CO}$ in the gas phase after assuming LTE (e.g., Section 6.1 in Palla \& Stahler 2004; Scoville et al. 1986). Under this approximation, an estimate for the column density of $\mathrm{CO}$ can be obtained after knowing the gas excitation temperature, the source brightness temperature (or upper limit), and line width, plus some characteristics of the specific molecular transition. Assuming a line FWHM of $\sim 1.9 \mathrm{~km} \mathrm{~s}^{-1}$ given by the Keplerian velocity field at $R_{\text {in }} \approx 30 \mathrm{AU}$ projected along the line of sight for a disk with inclination of $\approx 20 \mathrm{deg}$, and uniform excitation temperature of $50 \mathrm{~K}$ throughout the disk, we derived a $3 \sigma$ upper limit for the mass of gaseous $\mathrm{CO}$ of $M_{\mathrm{CO}}<1.9 \times 10^{-6} M_{\oplus}$. For an excitation temperature of $100 \mathrm{~K}$, this upper limit would become $\approx 3.6 \times 10^{-6} M_{\oplus}$. If we consider a radially dependent excitation temperature equal to the dust temperature radial profile as constrained from our analysis for small $(\approx 5 \mu \mathrm{m})$ or large $(\approx 1 \mathrm{~mm})$ particles, we derive upper limits of $\approx 1.6 \times 10^{-6} M_{\oplus}$ and $\approx 1.2 \times 10^{-6} M_{\oplus}$, respectively. These upper limits in $\mathrm{CO}$ mass are a factor of a few more sensitive than the value derived 


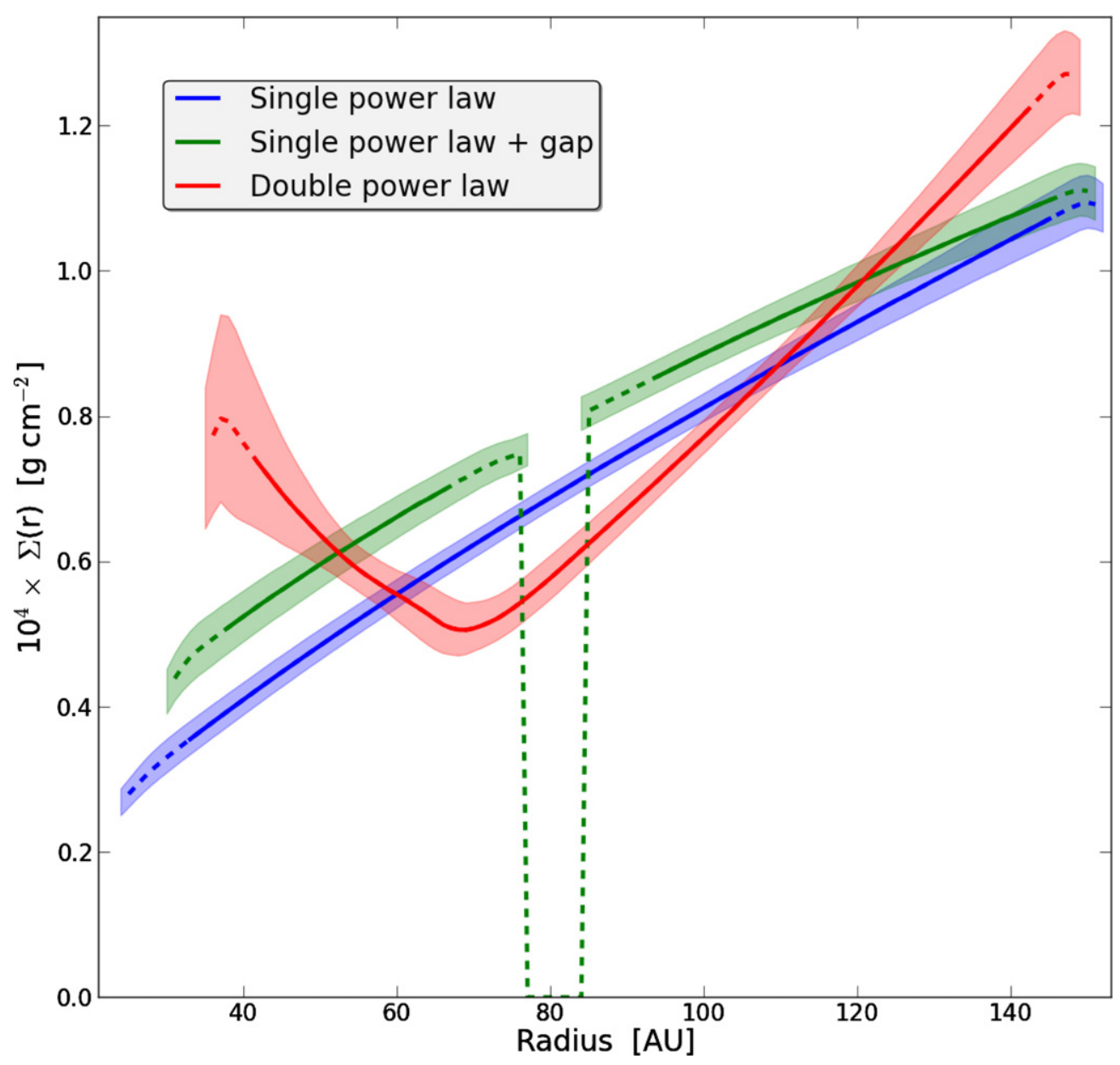

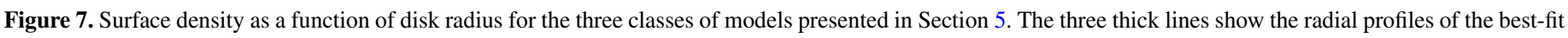

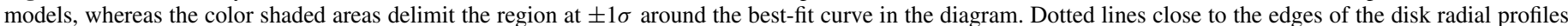
reflect the uncertainties on the inner and outer disk radii, as well as on the radii of the gap edges.

by the non-detection of the ${ }^{12} \mathrm{CO}(J=3-2)$ rotational line with SCUBA (Najita \& Williams 2005), after correcting for the different disk inclination and outer radius used by those authors.

The best-fit models for the three classes of disk models presented in Section 5 have a dust mass of $\approx 0.2 M_{\oplus}$. The $3 \sigma$ upper limit we derived for the CO-to-dust mass ratio in the HD 107146 debris disk is therefore $<10^{-5}$. This is a factor of $3 \times\left(10^{2}-10^{4}\right)$ lower than the range of CO-to-dust mass ratios estimated for a sample of 15 primordial disks surrounding lowmass stars with ages of $\approx 1-10$ Myr (Williams \& Best 2014 and references therein). Relative to the $\mathrm{CO}$-to-dust mass ratio estimated in debris disks around A-type stars where $\mathrm{CO}$ gas has been detected, our upper limit for HD 107146 is lower by factors of $\approx 40,10^{3}, 7 \times 10^{4}$ than in $\beta$ Pic (age $\sim 10 \mathrm{Myr}$; Dent et al. 2014), 49 Ceti (age 10 Myr; Hughes et al. 2008; Zuckerman et al. 1995), and HD 21997 (age 30 Myr; Kospal et al. 2013; Moor et al. 2013), respectively.

\section{DISCUSSION}

Our analysis of the ALMA data provide the most stringent constraints on the physical structure of the HD 107146 debris disk at millimeter wavelengths to date. The results of our modeling show that at $1.25 \mathrm{~mm}$ the disk extends from about $30 \mathrm{AU}$ to $150 \mathrm{AU}$, with a decrease in the dust surface density around $\sim 70-80$ AU from the star, and with a surface density toward the outer radius of the disk which is comparable, or even larger than in the inner disk.

\subsection{A Gap in the Disk?}

As described in Section 5.3 the current data do not constrain the detailed radial profile of $\Sigma(r)$ in the region of the depletion. In Section 5 we described the constraints to the model parameters in the case of single power-law models with a gap as well as for double power-law models. Both classes of models provide a better fit to the ALMA data than single power-law models with no gap. A visual representation of the constrained radial profiles of the dust surface density for each of the three classes of models is shown in Figure 7.

If a gap is present in the HD 107146 disk then the most natural explanation would be the dynamical clearing by a planet. An alternative explanation involving "photoelectric instability," which has been proposed to reproduce rings in debris disks with detected gas (Lyra \& Kuchner 2013) is made unlikely by our very tight upper limit to the CO-to-dust mass ratio (Section 7): this mechanism can be efficient only for disks with gas-to-dust mass ratios larger than $\sim 1$, which would require a high value $>10^{5}$ for the gas-to-CO mass ratio. The relatively old age of the star, i.e., $\sim 80-200 \mathrm{Myr}$, suggests that the gas-to-CO mass ratio in the system is probably lower than $\sim 10^{5}-10^{4}$. This is the range of values estimated for the younger TW Hya primordial disk (Favre et al. 2013), where nearly all the gas is under the form 
of molecular $\mathrm{H}_{2}$ that gets efficiently dispersed by the process of photo-evaporation within the first $\sim 10 \mathrm{Myr}$ after the formation of the star. However, the gas-to-CO ratio may be underestimated if molecular $\mathrm{CO}$ has been strongly depleted, either because of condensation onto dust grains and/or UV photodissociation (e.g., Dent et al. 1995; Kamp \& Bertoldi 2000). The temperature derived by our models for small dust particles across the disk is always $\gtrsim 30 \mathrm{~K}$, indicating that $\mathrm{CO}$ condensation onto grains should not be effective, at least as long as grains are surrounded by a CO substrate (see the discussion in Najita \& Williams 2005). As for the UV photodissociation, given the relatively late spectral type of HD 107146, most dissociating UV photons are expected to come from the interstellar radiation field rather than from the central star (Kamp \& Bertoldi 2000; Greaves et al. 2000). Najita \& Williams (2005) applied photodissociation models to HD 107146 and estimated a gas-to-dust mass ratio $<1$ even in the case of a significant amount of molecular $\mathrm{H}_{2}$ still present in the system. However, the geometry and temperature of the putative gas in the debris disk is obviously uncertain and can potentially play an important role in those calculations.

In the scenario of a planet-induced gap, a measurement of the gap width provides an estimate for the mass of the putative planet. At a given distance from the central star, a more massive planet carves a wider gap (Quillen 2006), which is set by the region of overlapping mean motion resonances on either side of the planet (Wisdom 1980). When the resonance widths exceed the distance between them, particle motion becomes chaotic. If the solids in the disk are in nearly circular orbits and the planet has low eccentricity, then the chaotic zone boundary is given by $d a_{z} \approx(1.3-2.0) \times \mu^{2 / 7}$, where $d a_{z}$ is the difference between the zone edge semi major axis and that of the planet divided by the semi major axis of the planet, and $\mu$ is the ratio between the planet and stellar mass. The range of (1.3-2.0) for the possible normalization coefficients has been obtained by numerical simulations incorporating collisions in a diffusive limit (Quillen \& Faber 2006; Chiang et al. 2009). However, Gladman (1993) has demonstrated that solids in the outer regions of a chaotic zone will stay bound in that region even with their chaotic dynamics. Solids which are closer to the planet, within a region called crossing zone, will instead cross the orbit of the planet and get scattered out very efficiently. The size of the crossing region, which should be more directly related to the notion of a gap in solids around the planet, is given by $d a_{z} \approx(2.1-2.4) \times \mu^{1 / 3}$.

Under the framework of the models with the surface density described as a single power law with gap, $d a_{z} \approx \Delta R_{\text {gap }} /\left(2 R_{\text {gap }}\right)$. The mass of the putative planet in HD 107146 can therefore be estimated using the values of $\Delta R_{\text {gap }}$ and $R_{\text {gap }}$ derived by our analysis and reported in Table 2 . We obtain $6.1_{-3.1}^{+2.1} M_{\oplus}$ and $4.1_{-2.1}^{+1.4} M_{\oplus}$ for values of 2.1 and 2.4 for the normalization coefficient in the $d a_{z}-\mu$ relation for the crossing zone, respectively. The uncertainties on these estimates are derived from propagating the uncertainties on $\Delta R_{\text {gap }}$ and $R_{\text {gap }}$. If we used the relation from the chaotic zone theory, we would obtain $5.3_{-3.1}^{+2.2} M_{\oplus}$ and $1.2_{-0.7}^{+0.5} M_{\oplus}$ for values of 1.3 and 2.0 for the normalization coefficient, respectively. A value of $\approx 1.9 M_{\oplus}$ is obtained from the results of recent numerical calculations which account for disruptive collisions other than the gravitational interaction between a planet and a ring of planetesimals (Nesvold \& Kuchner 2014).

Although only more sensitive and higher angular resolution observations can possibly confirm the presence of a gap in the HD 107146 debris disk, this analysis shows the potential of probing (indirectly) with ALMA the presence of terrestrial planets (and more massive planets) at wide separations and embedded in planetesimal disks. Terrestrial planets are still well beyond the reach of direct imaging techniques. In the case of HD 107146, upper limits of $\sim 10-13 M_{\text {Jup }}$ at distances $\gtrsim 15$ AU from the central star have been obtained by highcontrast imaging in the near IR (Apai et al. 2008; Metchev \& Hillenbrand 2009; Janson et al. 2013).

\subsection{Origin of the HD 107146 Debris Disk}

An interesting result of our analysis is that, at least in the outer regions of the HD 107146 debris disk, the dust surface density increases with the distance from the central star. A similar behavior has been recently found for the debris disk around the M-type, $\approx 10$ Myr-old young star AU Mic (MacGregor et al. 2013). In the case of HD 107146, single power-law models with or without a gap predict power-law indices for the dust surface density radial profile of $\approx 0.57$ and 0.75 , respectively, with uncertainties lower than 0.1 . In the case of double powerlaw models, the power-law index beyond $R_{\text {break }} \approx 70 \mathrm{AU}$ is about 1.3. This behavior is different from all the younger gasrich primordial disks in which the dust surface density decreases with radius $^{7}$ (Andrews \& Williams 2007; Andrews et al. 2009; Isella et al. 2009; Guilloteau et al. 2011).

The millimeter-sized grains traced by ALMA are insensitive to the stellar radiation pressure and trace the location of the planetesimals that produced them by collision. Since planetesimals are formed in primordial disks where surface densities and collisional rates decrease with radius, it is unnatural to think that this feature is an imprint of initial conditions. Rather, it may reflect collisional evolution and depletion of planetesimals with radius.

In the models of icy planets formation by Kenyon \& Bromley $(2002,2008)$ the dust in debris disks is efficiently produced once a population of Pluto-sized objects with radii of $\sim 1000 \mathrm{~km}$ or larger is formed in the disk. These bodies stir smaller leftover planetesimals to disruption velocities, so that the outcome of their collisions is a very efficient production of debris dust particles. Once $\sim 1-10 \mathrm{~km}$ sized planetesimals have been ground, the production of dust is inhibited and the dust production rate decreases significantly. Therefore, in these models dust traces the recent formation of a population of Pluto-sized objects triggering the collisional cascade.

These Pluto-sized objects are formed at different times across the disk, so that the spatial variation of dust surface density can probe how the formation of icy planets propagates throughout the disk. For collisional processes, the growth time of solids in the disk is $t_{\text {growth }} \propto P / \Sigma$ where $P$ is the orbital period (Lissauer 1987) and $\Sigma$ the surface density of solids. As $P \propto r^{3 / 2}$, if the initial surface density $\Sigma$ of solids decreases with radius (or increases with a profile shallower than a power law with index $3 / 2$ ), then solids growth is faster closer to the star. For a debris disk extending between $\sim 30$ and $\sim 150$ AU with the mass in dust particles as estimated for HD 107146 at $\sim 100 \mathrm{Myr}$, the Kenyon $\&$ Bromley (2008) models predict a timescale for the formation of $1000 \mathrm{~km}$ planetesimals of just $\sim 10$ Myr in the inner disk (30 AU), but as large as $\sim 1.4 \mathrm{Gyr}$ in the outer disk (150 AU).

\footnotetext{
7 An exception is for transitional disks which present inner regions partially or fully depleted in dust. In the inner regions of these disks the dust surface density increases with radius, although with very steep radial profiles. In the outer regions the dust surface density decreases with radius similar to the case of non-transitional primordial disks (Andrews et al. 2011; Isella et al. 2012).
} 
Taken at face value, the HD 107146 system would have had enough time to form Pluto-sized objects in the innermost disk regions, but not in the outermost ones, the furthest $1000 \mathrm{~km}$ objects being formed at $\sim 57,62,78$ AU for HD 107146 ages of 80, 100, and $200 \mathrm{Myr}$, respectively. If Pluto-sized objects are not formed in the outer regions, dust production via collisional cascade would not be efficient in those regions, contrary to our result of a dust surface density which increases with radius in the outer disk.

These estimates strongly depend on the initial conditions assumed for the distribution of solids at the beginning of the calculation. ${ }^{8}$ In particular, the timescale for the formation of Pluto-sized objects scales with the density of solids with a power of $-1.15 .^{9}$ The Kenyon \& Bromley (2008) models adopt an initial surface density of solids with radii of $\sim 1-1000 \mathrm{~m}$ with a power law radial profile with index $-3 / 2$ and a surface density at $30 \mathrm{AU}$ of $\approx 0.1-0.2 \mathrm{~g} \mathrm{~cm}^{-2}$ comparable to the distribution of solids in the minimum-mass solar nebula model (MMSN; Weidenschilling 1977; Hayashi 1981). In order to decrease the timescale for the formation of Pluto-sized objects in the outer disk down to values lower than the estimated age of HD 107146, i.e., $\lesssim 200 \mathrm{Myr}$, the initial density has to be increased by a factor of $\sim 10$. Two possible ways to achieve this are by increasing the solids density at all radii by this factor, or by making the radial profile nearly flat, i.e., $\Sigma_{i} \propto r^{-0.1}$, while keeping the same density in the inner disk.

In the former case the total mass of these solids would be increased by a factor of 10 , giving a mass in 1-1000 $\mathrm{m}$ bodies of $\sim 4.7 \times 10^{30} \mathrm{~g} \approx 800 M_{\oplus}$, while in the latter the required mass is $\sim 2 \times 10^{30} \mathrm{~g} \approx 340 M_{\oplus}$. These values are close to the masses in smaller solids (radii $\lesssim 1 \mathrm{~cm}$ ) estimated for the brightest disks in nearby, $\approx 1 \mathrm{Myr}$ old star forming regions (e.g., Andrews \& Williams 2005, 2007; Isella et al. 2009; Ricci et al. 2010a, 2010b; Andrews et al. 2013). If at this early stage of disk evolution the gas-to-solid mass ratio is still similar to the value $\sim 100$ as found in the interstellar medium (ISM), the required initial gas mass is in the range $\approx 0.10-0.25 M_{\odot}$. This roughly corresponds to the mass limit for having gravitational instabilities in a disk around a $1 M_{\odot}$ star such as HD 107146 (e.g., Boss 1997; Rice et al. 2005).

Our analysis shows that if the primordial disk around HD 107146 was initially very massive and close to the gravitational instability limit, models of solid growth predict that the formation of Pluto-sized objects could have had the time to propagate outward to the disk outermost regions. The inferred rising surface density of small solids could be the result of this propagation, the outer regions containing more dust grains than the inner ones because having initiated the collisional cascade more recently.

Kennedy \& Wyatt (2010) use this idea of a "self-stirred" planetesimal belt to predict the surface density of solids across the disk. They divide their simulations in two cases, depending on whether the collisional timescale $t_{\mathrm{c}}$ is lower or larger than the time at which the stirring is initiated, $t_{\text {stir }}$. For models in which $t_{\mathrm{c}} \ll t_{\text {stir }}$, the evolution of solids is violent and leads to rising, very steep surface densities with $p>2$, much larger

\footnotetext{
8 Instead, for disks with ages $\gg 10 \mathrm{Myr}$, like in the case of HD 107146, dust production rates and dust masses are nearly insensitive to the specific choice for the fragmentation parameters (see discussion in Kenyon \& Bromley 2008, Section 3.3.2).

9 Note that this is slightly different from the inverse dependence that would be inferred from the simple $t_{\text {growth }} \propto P / \Sigma$ argument; this discrepancy is due to the effect of gas drag in the early phases of planetesimal growth in the primordial disk (see Kenyon \& Bromley 2008).
}

than the value constrained for HD 107146. If instead $t_{\mathrm{c}}>t_{\mathrm{stir}}$, consistently with the case of HD 107146 after using the Kennedy $\&$ Wyatt fiducial parameter values and a stellar mass of $1 M_{\odot}$ (see Equation (10) in their paper), the evolution is slower and this results into a more shallower but still rising surface density radial profile. For the model shown on the left panel of Figure 2 in Kennedy \& Wyatt (2010), at an age of 100 Myr the surface density shows a rather moderate increase with radius in the disk outer regions, which is more consistent with the results of our analysis on HD 107146. Another interesting aspect of these models is that right after the radius at which the surface density peaks, the surface density rapidly drops by orders of magnitude. This can explain the absence in Figure 5 of any significant residuals beyond the outer radius $R_{\text {out }}$ of our models. A more thorough theoretical investigation adapted specifically to the characteristics of the HD 107146 system is needed to further test models of debris disks produced by the self-stirring of a planetesimal belt.

Another possible scenario to explain a rising surface density with radius involves dynamical interaction between a planetesimal belt and a fully formed planetary system. According to the Nice model, dynamical gravitational interaction between the giant planets and the young Kuiper belt has played a crucial role in shaping the current architecture of the solar system (Gomes et al. 2005; Morbidelli et al. 2005). After several hundreds of megayears from the dispersal of the gas-rich primordial disks, Neptune migrated into the Kuiper belt and dynamically excited the orbit of several Kuiper belt objects, possibly explaining the late heavy bombardment (LHB) of the Moon (Tera et al. 1974). Because of this interaction, a large number of planetesimals got scattered both inward and outward spreading out the distribution of mass. The rate at which planetesimals were scattered inward is much greater than the rate at which they were scattered outward. As a result, the surface density profile got asymmetric with radius, with the bulk of the mass being represented by a surface density with a relatively shallow and rising slope $\left(\Sigma \propto r^{1.5}\right)$ followed by a very steep falloff $\left(\Sigma \propto r^{-5}\right)$ at larger radii (Booth et al. 2009).

The scenarios described here is only one of the infinite possible outcomes of a planetary system-planetesimal belt interaction. It is referred to the case of the solar system, and therefore does not reproduce in detail the characteristics observed for the HD 107146 disk (see Section 8.3). At the same time this example shows the potential of this mechanism to possibly reproduce a relatively shallow surface density of small particles that is increasing with radius. Whether this scenario can explain the main properties of the HD 107146 remains to be tested. Models that account for both the dynamical interaction between a planetesimals belt and a planetary system as well as the debris dust production through the collision of large bodies are needed (e.g., Nesvold et al. 2013). The presence of planets in the system will be investigated through future high angular resolution observations (see discussion above).

We note that an important underlying assumption of our models is that the dust opacity is the same throughout the disk. The result of a dust surface density that is increasing with radius could be modified if the dust opacity $\kappa_{v}$ at the wavelength of the ALMA observations were a function of the location in the disk. Since $I_{1.25 \mathrm{~mm}}(r) \propto T(r) \times \Sigma(r) \times \kappa_{1.25 \mathrm{~mm}}(r)$, the terms $\Sigma(r)$ and $\kappa_{1.25 \mathrm{~mm}}(r)$ would be degenerate. This means that our ALMA data could in principle be reproduced also by disk models with a $\kappa_{1.25 \mathrm{~mm}}(r)$ function that is increasing with radius and with a dust surface density that is flat or even decreasing with the distance from the central star. 
Spatial variations of the dust opacity could reflect variations of the dust chemical composition and/or grain physical morphology, as well as variations in the grain size distribution. For example, if the slope $q$ of the grain size distribution were changing from 3.0 in the inner disk to 3.5 in the outer disk, that would produce a variation of a factor of $\sim 3$ in $\kappa_{1.25 \mathrm{~mm}}$. That is close to the variation of the dust surface density between the inner and outer regions of the disk, as constrained by our models assuming a constant $\kappa_{1.25 \mathrm{~mm}}$ (see Figure 7 ). The only way to break the degeneracy between $\Sigma(r)$ and $\kappa_{v}(r)$ and better constrain the radial profile of the dust surface density is by observing the disk at high angular resolution at multiple wavelengths in the (sub-)millimeter, as done for primordial disks (Isella et al. 2010; Guilloteau et al. 2011; Perez et al. 2012; Trotta et al. 2013). Future ALMA observations at multiple wavelengths have the potential to do this in the case of HD 107146.

\subsection{Comparison with the Kuiper Belt}

The HD 107146 main sequence star has the same spectral type and luminosity class as the Sun. Its younger age allows us to investigate the properties of a planetesimal belt around a young Sun-like star.

While thermal emission from solids in extra-solar planetesimal belts trace small dust particles with sizes $\lesssim 1 \mathrm{~cm}$, emission from dust produced in the Kuiper belt has not been detected yet and only large, kilometer-sized bodies can be seen in the Kuiper belt. The only way to attempt a comparison between the solar system's Kuiper belt and extra-solar belts is via an observational characterization of large solids and small dust particles in the former and latter systems respectively, using models for the dynamics and physics of collisions to connect the information on solids with very different sizes.

The vast majority of objects discovered in the solar system with a greater average distance than Neptune ("trans-Neptunian objects," TNOs) have a semi-major axis between $\approx 30$ and $50 \mathrm{AU}$. These TNOs constitute the so-called "classical Kuiper belt." Whereas the inner radius of the classical Kuiper belt is very similar to that constrained for HD 107146, the outer disk spreads out a factor of $\sim 3$ further.

Several TNOs have also been found beyond the classical Kuiper belt, as far as $\sim 1000 \mathrm{AU}$ from the Sun, therefore at distances even larger than the outer radius of the HD 107146 debris disk. These objects are characterized by relatively large eccentricities and inclinations to the ecliptic. They have likely been scattered out through gravitational interactions with the giant planets rather than being formed in situ, and for this reason are called "scattered disk objects" (SDOs).

Vitense et al. (2010) analyzed a large sample of TNOs known at the time and used an algorithm to remove the observational biases due to the inclination and distance selection effects and estimated the main parameters of the Kuiper belt. They derived a mass of $\approx 0.12 M_{\oplus}$, half of which located in the classical Kuiper belt, the other half in SDOs. In order to predict the spatial distribution of dust particles in the presumed Kuiper belt debris disk, they applied to their "de-biased" Kuiper belt a numerical code that includes the effects of stellar gravity, radiation pressure, and Poynting-Robertson drag, as well as disruptive and erosive collisions. They derived a surface density of dust with a peak around $\approx 40 \mathrm{AU}$ with a shallow radial profile in the inner disk down to radii $\lesssim 20 \mathrm{AU}$, and a steeper falloff with a power-law index of $\approx-2.0$ out to radii of several hundreds AU.
In addition to having a very different radial profile of the dust surface density, the debris disk around HD 107146 has a very different mass than the estimated Kuiper belt debris disk. The mass of kilometer-sized bodies in the Kuiper belt estimated by Vitense et al. (2010) is within a factor of two from the mass in dust estimated for the HD 107146 debris disk. Since the mass of kilometer-sized bodies that generate the dust is much larger than the mass of the dust itself, this implies that the dust in the HD 107146 is much more massive than in the Kuiper belt, which is in line with the much younger age of the HD 107146 system.

Quantifying the mass of the planetesimal belt surrounding HD 107146 from the mass in dust is prone to large uncertainties because of the huge step in solid size. If we could extrapolate the grain size distribution with a power-law index of 3.25 as derived in Section 4.1 all the way to bodies with sizes of 100 and $1000 \mathrm{~km}$, we would find $M_{\text {belt }} \sim 10^{4}$ and $5 \times 10^{4} M_{\oplus}$, respectively. These values are unreasonably high, as they would require a mass in gas and solids for the parental primordial disk larger than the mass of the central star (assuming the canonical value of 100 for the gas-to-dust mass ratio), much larger than the masses estimated for primordial disks around $\sim 1$ Myr old premain-sequence stars (Andrews \& Williams 2005; Isella et al. 2009; Ricci et al. 2010a).

Lower, more realistic values are derived by considering a power law index of 3.6 for the size distribution of large bodies as derived by Vitense et al. (2010) in the case of the Kuiper belt. With this value the mass in bodies as large as $1000 \mathrm{~km}$ would be $100 M_{\oplus}$. This is only a factor of a few larger than the mass in planetesimals invoked by the Nice model to describe the scattering event that lead to the LHB and to the dispersal of the vast majority of planetesimals in the early solar system (e.g., Booth et al. 2009).

\section{SUMMARY}

We presented continuum and spectral line data for the HD 107146 debris disk using ALMA in Cycle 0 at a wavelength of about $1.25 \mathrm{~mm}$. These are the most sensitive, highest angular resolution observations carried out so far at millimeter wavelengths for HD 107146, a $100 \mathrm{Myr}$ old solar analog.

We analyze the ALMA interferometric visibilities for the dust continuum emission using debris disk models to investigate the radial distribution of debris dust particles. We used different functional forms to parameterize the radial profile of the dust surface density, namely a single power law, a single power law with a gap, and a double power law. The surface density of all these models is truncated at an inner and outer radius, respectively.

Our modeling shows that the dust in the disk extends from about 30 to $150 \mathrm{AU}$ from the central star. Disk models with $\mathrm{a} \approx 8 \mathrm{AU}$-wide gap at about $80 \mathrm{AU}$, as well as double powerlaw models with a decreasing surface density till about $70 \mathrm{AU}$ and increasing afterward better reproduce the ALMA data than single power-law models with no gap. The general result is that despite that the ALMA data cannot discriminate between single power-law models with gap and double power-law models, the HD 107146 debris disk shows a depletion of dust at about 70-80 AU, and a dust surface density toward the outer edge of the disk, which is comparable or larger than the values found in the inner disk. 
Future ALMA observations with better sensitivity as well as angular resolution will allow to better constrain the radial distribution of dust in the HD 107146 debris disk and distinguish between the possible scenarios presented here. If these future observations confirm the gap structure, a planet with a mass of $\approx$ a few Earth masses in a nearly circular orbit at $\sim 80$ AU from the central star would be a likely explanation for the presence of the gap.

The feature of a dust surface density that is increasing with radius in the outer regions of the disk can be qualitatively explained by self-stirring models of planetesimal belts in which the formation of Pluto-sized objects trigger disruptive collisions of large bodies and the production of debris dust. We showed that if the primordial disk that generated the HD 107146 system was a massive disk close to the self-gravitation limit, then the planetesimal belt would have had enough time to form $\sim 1000 \mathrm{~km}$ sized bodies in the outer disk regions, and they would then efficiently stir the planetesimals themselves.

Alternatively or in addition to this self-stirring mechanism, the distribution of planetesimals can be affected by the presence of one or more planets in the system. In order to test this scenario in the case of HD 107146, models that account for both the dynamical interaction between a planetesimals belt and a planetary system as well as the debris dust production through the collision of large bodies are needed. The presence of planets will be directly investigated through high-angular resolution observations with the new generation of high-contrast cameras (e.g., GPI, SPHERE) as well as with the future class of $30-40 \mathrm{~m}$ telescopes at optical and infrared wavelengths.

The modeling adopted here assumes a constant value for the dust opacity throughout the disk. The inferred radial profile of the dust surface density would change if the dust opacity were a function of the distance from the central star. Future ALMA observations at multiple wavelengths will allow us to quantify the spatial variation of dust opacity across the disk.

From our non-detection of the ${ }^{12} \mathrm{CO}(J=3-2)$ rotational emission line we derived a $3 \sigma$ upper limit of $1.9 \times 10^{-6} M_{\oplus}$ for the total mass of CO molecular gas. The upper limit of $10^{-6}$ obtained for the CO-to-dust mass ratio is about three to five orders of magnitude lower than younger primordial disks as well as some debris disks with detected $\mathrm{CO}$ emission around younger A-type stars.

This paper makes use of the following ALMA data: ADS/ JAO.ALMA\#2011.0.00470.S. ALMA is a partnership of ESO (representing its member states), NSF (USA), and NINS (Japan), together with NRC (Canada) and NSC and ASIAA (Taiwan), in cooperation with the Republic of Chile. The Joint ALMA Observatory is operated by ESO, AUI/NRAO, and NAOJ. J.M.C. acknowledges support from NSF grant AST-1109334. The National Radio Astronomy Observatory is a facility of the National Science Foundation operated under cooperative agreement by Associated Universities, Inc.

\section{REFERENCES}

Andrews, S. M., Rosenfeld, K. A., Kraus, A. L., \& Wilner, D. J. 2013, ApJ, 771,129

Andrews, S. M., \& Williams, J. P. 2005, ApJ, 631, 1134

Andrews, S. M., \& Williams, J. P. 2007, ApJ, 659, 705

Andrews, S. M., Wilner, D. J., Espaillat, C., et al. 2011, ApJ, 732, 42

Andrews, S. M., Wilner, D. J., Hughes, A. M., Qi, C., \& Dullemond, C. P. 2009, ApJ, 700, 1502

Apai, D., Janson, M., Moro-Martn, A., et al. 2008, ApJ, 672, 1196

Ardila, D. R., Golimowski, D. A., Krist, J. E., et al. 2004, ApJ, 617L, 147
Booth, M., Wyatt, M. C., Morbidelli, A., Moro-Martin, A., \& Levison, H. F 2009, MNRAS, 399, 385

Boss, A. P. 1997, Sci, 276, 1836

Burns, J. A., Lamy, P. L., \& Soter, S. 1979, Icar, 40, 1

Carpenter, J. M., Bouwman, J., Silverstone, M. D., et al. 2008, ApJS, 179, 423

Carpenter, J. M., Wolf, S., Schreyer, K., Launhardt, R., \& Henning, Th. 2005, AJ, 129, 1049

Chiang, E., Kite, E., Kalas, P., Graham, J. R., \& Clampin, M. 2009, ApJ, 693, 734

Corder, S., Carpenter, J. M., Sargent, A. I., et al. 2009, ApJ, 690L, 65

Dent, W. R. F., Greaves, J. S., Mannings, V., Coulson, I. M., \& Walther, D. M. 1995, MNRAS, 277, L25

Dent, W. R. F., Wyatt, M. C., Roberge, A., et al. 2014, Sci, 343, 1490

Dohnanyi, J. S. 1969, JGR, 74, 2531

Draine, B. T. 2006, ApJ, 636, 1114

Ertel, S., Wolf, S., Metchev, S., et al. 2011, A\&A, 533, 132

Favre, C., Cleeves, L. I., Bergin, E. A., Qi, C., \& Blake, G. A. 2013, ApJL, 776, L38

Foreman-Mackey, D., Hogg, D. W., Lang, D., \& Goodman, J. 2013, PASP, 125,306

Gaspar, A., Psaltis, D., Rieke, G. H., \& Ozel, F. 2012, ApJ, 754, 74

Gladman, B. 1993, Icar, 106, 247

Gomes, R., Levison, H. F., Tsiganis, K., \& Morbidelli, A. 2005, Natur, 435, 466

Goodman, J., \& Weare, J. 2010, Comm. App. Math. Comp. Sci., 5, 65

Greaves, J. S., Coulson, I. M., \& Holland, W. S. 2000, MNRAS, 312, L1

Guilloteau, S., Dutrey, A., Pitu, V., \& Boehler, Y. 2011, A\&A, 529, 105

Hayashi, C. 1981, PThPS, 70, 35

Hillenbrand, L. A., Carpenter, J. M., Kim, J. S., et al. 2008, ApJ, 677, 630

Hughes, A. M., Wilner, D. J., Andrews, S. M., et al. 2011, ApJ, 740, 38

Hughes, A. M., Wilner, D. J., Kamp, I., \& Hogerheijde, M. R. 2008, ApJ, 681,626

Isella, A., Carpenter, J. M., \& Sargent, A. I. 2009, ApJ, 701, 260

Isella, A., Carpenter, J. M., \& Sargent, A. I. 2010, ApJ, 714, 1746

Isella, A., Perez, L., \& Carpenter, J. M. 2012, ApJ, 747, 136

Janson, M., Brandt, T. D., Moro-Martn, A., et al. 2013, ApJ, 773, 73

Kamp, I., \& Bertoldi, F. 2000, A\&A, 353, 276

Kennedy, G. M., \& Wyatt, M. 2010, MNRAS, 405, 1253

Kenyon, S. J., \& Bromley, B. C. 2002, ApJ, 577L, 35

Kenyon, S. J., \& Bromley, B. C. 2008, ApJS, 179, 451

Kospal, A., Moor, A., Juhasz, A., et al. 2013, ApJ, 776, 77

Lissauer, J. J. 1987, Icar, 69, 249

Lyra, W., \& Kuchner, M. 2013, Natur, 499, 184

MacGregor, M. A., Wilner, D. J., Rosenfeld, K. A., et al. 2013, ApJ, 762L, 21

Matthews, B. C., Kennedy, G., Sibthorpe, B., et al. 2014, in Protostars \& Planets VI, ed. H. Beuther, R. Klessen, C. Dullemond, \& Th. Henning (Tucson, AZ: Univ. Arizona Press), in press, arXiv:1401.0743

McMullin, J. P., Waters, B., Schiebel, D., Young, W., \& Golap, K. 2007, in ASP Conf. Ser. 376, Astronomical Data Analysis Software and Systems XVI, ed. R. A. Shaw, F. Hill, \& D. J. Bell (San Francisco, CA: ASP), 127

Metchev, S. A., \& Hillenbrand, L. A. 2009, ApJS, 181, 62

Miyake, K., \& Nakagawa, Y. 1993, Icar, 106, 20

Moor, A., Abraham, P., Derekas, A., et al. 2006, ApJ, 644, 525

Moor, A., Juhasz, A., Kospal, A., et al. 2013, ApJL, 777, L25

Morales, F. Y., Rieke, G. H., Werner, M. W., et al. 2011, ApJL, 730, L29

Morbidelli, A., Levison, H. F., Tsiganis, K., \& Gomes, R. 2005, Natur, 435, 462

Najita, J., \& Williams, J. P. 2005, ApJ, 635, 625

Nesvold, E. R., \& Kuchner, M. J. 2014, ApJ, in press

Nesvold, E. R., Kuchner, M. J., Rein, H., \& Pan, M. 2013, ApJ, 777, 144

Palla, F., \& Stahler, S. W. 2004, The Formation of Stars (Cambridge: Wiley$\mathrm{VCH})$

Pan, M., \& Schlichting, H. E. 2012, ApJ, 747, 113

Perez, L. M., Carpenter, J. M., Chandler, C. J., et al. 2012, ApJ, 760L, 17

Pollack, J. B., Hollenbach, D., Beckwith, S., et al. 1994, ApJ, 421, 615

Quillen, A. C. 2006, MNRAS, 372, L14

Quillen, A. C., \& Faber, P. 2006, MNRAS, 373, 1245

Ricarte, A., Moldvai, N., Hughes, A. M., et al. 2013, ApJ, 774, 80

Ricci, L., Testi, L., Maddison, S. T., \& Wilner, D. J. 2012, A\&A, 539L, 6

Ricci, L., Testi, L., Natta, A., et al. 2010a, A\&A, 512, 15

Ricci, L., Testi, L., Natta, A., \& Brooks, K. J. 2010b, A\&A, 521, 66

Rice, W. K. M., Lodato, G., \& Armitage, P. J. 2005, MNRAS, 364L, 56

Roccatagliata, V., Henning, Th., Wolf, S., Rodmann, J., et al. 2009, A\&A, 497, 409

Schneider, G., Grady, C. A., Hines, D. C., et al. 2014, AJ, 148, 59

Scoville, N. Z., Sanders, D. B., Sargent, A. I., et al. 1986, ApJ, 311L, 47

Silverstone, M. D. 2000, PhD thesis, Univ. California, Los Angeles

Tera, F., Papanastassiou, D. A., \& Wasserburg, G. J. 1974, E\&PSL, 22, 1 
Trotta, F., Testi, L., Natta, A., Isella, A., \& Ricci, L. 2013, A\&A, 558,64

Valenti, J. A., \& Fischer, D.A. 2005, ApJS, 159, 141

van Leeuwen, F. 2007, A\&A, 474, 653

Vitense, Ch., Krivov, A. V., \& Lohne, T. 2010, A\&A, 520, 32

Warren, S. G. 1984, ApOpt, 23, 1206

Weidenschilling, S. J. 1977, Ap\&SS, 51, 153

Weingartner, J. C., \& Draine, B. T. 2001, ApJ, 548, 296

Williams, J. P., \& Best, W. M. J. 2014, ApJ, 788, 59
Williams, J. P., Najita, J., Liu, M. C., et al. 2004, ApJ, 604, 414

Wilner, D. J., \& Welch, W. J. 1994, ApJ, 427, 898

Wisdom, J. 1980, AJ, 85, 1122

Wyatt, M. C. 2006, ApJ, 639, 1153

Wyatt, M. C. 2008, ARA\&A, 46, 339

Zubko, V. G., Mennella, V., Colangeli, L., \& Bussoletti, E. 1996, MNRAS, 282, 1321

Zuckerman, B. 2001, ARA\&A, 39, 549

Zuckerman, B., Forveille, T., \& Kastner, J. H. 1995, Natur, 373, 494 